\title{
Rising above the rhetoric: mobile applications and the delivery of cost-effective cardiovascular care in resource-limited settings
}
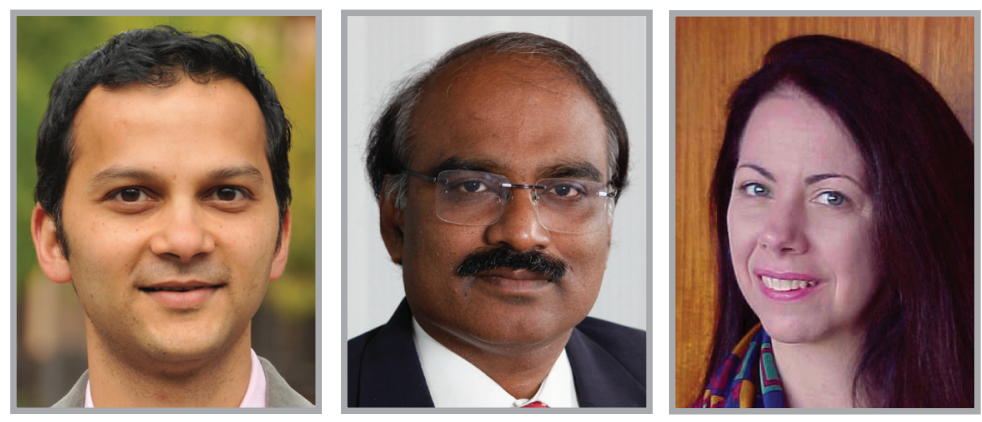

Dhruv S Kazi ${ }^{* 1,2,3}$, Dorairaj Prabhakaran ${ }^{4}$ \& Ann F Bolger ${ }^{1,2}$

The rising burden of cardiovascular disease (CVD) poses a critical threat to the communities and economies of low- and middle-income countries (LMICs) [1]. Several large studies have conclusively shown that CVD in LMICs strikes individuals a decade earlier than in high-income countries, often killing or disabling patients at the peak of their economic productivity $[2,3]$. The rapid increase in the prevalence of risk factors for CVD, such as tobacco consumption, diabetes mellitus, and hypertension, coupled with weak health systems that offer limited access to medications, revascularization technologies, and longterm care conspire to produce an agestandardized mortality from CVD that is substantially higher in LMICs than in high-income countries.

Recent years have seen the global community recognize the catastrophic implications of failing to effectively address the CVD epidemic. In 2012, following on from the United Nations High-Level Meeting on Non-Communicable Diseases (NCDs) from the previous year, the World Health Organization urged member states to reduce premature mortality from NCDs by $25 \%$ by 2025 . This will not be an easy undertaking: there are several barriers to addressing the epidemic of NCDs such as CVD in LMICs. Given the coexistence of pretransitional communicable diseases such as tuberculosis, malaria, maternal and childhood undernutrition and the epidemic of HIV in several LMICs, there is a lack of political will to allocate their scarce resources to the prevention and treatment of CVD. Indeed, some experts have argued that it would be iniquitous to mainstream the agenda of NCD prevention in LMICs [4]. Finally, cost is not the only barrier to CVD control in LMICs - even if all of the needed hospitals, clinics and catheterization laboratories were constructed, it would be challenging to provide trained staff to

\section{KEYWORDS}

- cardiovascular disease • cell phone $\bullet$ economics $\bullet$ mobile phone - LMIC • m-health • prevention

- resource-scarce settings

\section{"Recent years have seen the global community recognize the catastrophic implications of failing to effectively address the CVD epidemic."}


"Although the majority of these are older feature phones that are unable to run contemporary applications, smartphone penetration is rising rapidly; in 2013, more smartphones were sold in India than in the USA." run them. In 2014, the countries with the highest age-adjusted burden of CVD had fewer than ten physicians per 10,000 people (India has six physicians per 10,000 people, compared with 25 per 10,000 in the USA or 28 per 10,000 in the UK) [5]. The statistics on trained nursing and auxiliary staff are even more dismal. Clearly, reproducing the American or European healthcare models in LMICs is not a viable option. Addressing the CVD epidemic in LMICs will require innovative, out-of-the-box solutions that meet three key criteria to maximize impact: cost-effectiveness, as demonstrated in real-world settings in the context of LMICs [1]; scalability to national or international levels after initial success in smaller test settings [2]; and sustainability through private or public funding beyond the initial testing period [3].

The use of mobile technologies to address healthcare needs ( $m$-health) has the potential to transform healthcare delivery in resource-limited settings. The past decade has seen an explosion in mobile connectivity even in the most remote locations on the planet - the commercial mobile network covers $80 \%$ of the world's population and extends far beyond the electrical grid or the availability of improved sanitation. The example of India is illustrative: in a country of 1.2 billion people, there are 886 million cell phone subscriptions [6], and nearly every adult has access to one. Although the majority of these are older feature phones that are unable to run contemporary applications, smartphone penetration is rising rapidly; in 2013, more smartphones were sold in India than in the USA. The arrival in the market of high-quality, low-cost smartphones is likely to accelerate the pace of smartphone adoption in LMICs, even as intense price competition among mobile providers will continue to drive down the costs of voice and data services. It is predicted that by the end of the next decade, the majority of adults in LMICs will be carrying the equivalent of a minicomputer in their pockets and have high-quality access to the internet, opening up the possibility of disruptive innovation in the healthcare space. The writing is already on the wall: there were more than 43,000 health-related apps in the Apple App Store and more than 33,000 such apps on Google Play in 2013. Last year, mHealth attracted US\$564 million in venture capital investments. Clearly, this field is poised for exponential growth.

Mobile phones have the potential to address several pain points in healthcare delivery $[7]$. At their simplest, they serve as effective communication channels. This is no mean feat in settings where provider-patient communication would otherwise require a visit to a local bricks-andmortar clinic that may be many miles (and many hours) away and may not be best equipped to handle the acuity of the patient's complaints. A phone- or text message-based service can help triage patients quickly and inexpensively, followup patients who have been recently discharged from the hospital or provide reminders for medication adherence. For instance, applications can inexpensively and expeditiously collect patient data for follow-up (e.g., using text- or voicebased systems to create information systems that can help measure and improve healthcare quality), improve work flow (e.g., with text-based appointment reminders), assist with human resource management (e.g., for managing providers in a healthcare system) or help manage financial data (e.g., for hospitals). These services that focus on communication and data curation can be considered as 'mHealth 1.0' (borrowing the syntax used to grade commercial web applications) - technologically rudimentary but nevertheless clinically immensely valuable. For instance, simply being able to measure medication adherence may lead to innovative interventions for improving long-term adherence in the future. The majority of the currently available apps reside in this ' $\mathrm{mHealth} 1.0$ ' space.

One level up are mHealth solutions that collect data directly through embedded sensors ('mHealth 1.5'). This would include a blood pressure cuff or a glucometer that automatically uploads patient-identified data to a central repository. Such solutions are already available for personal use in high-income countries, but information technology barriers in these settings have impeded their integration into electronic health records. Given that many LMICs are moving towards the adoption of open-source electronic health records, they may 'leapfrog' wealthier nations in their ability to seamlessly integrate sensor-acquired data in the near future. This would be an important advance - systematically measuring and monitoring blood sugar and blood pressure is currently an expensive undertaking in rural, low-resource settings and is a major barrier to the effective control of hypertension and diabetes.

Researchers are increasingly interested in apps that can help improve decision-making, almost all of which are directed at providers rather 
than patients. Decision support applications ('mHealth 2.0') have been tested in a variety of settings, particularly in the field of maternal and child health, where the most robust applications of this nature have been created to date. A typical decision support software will prompt the user - usually a community health worker or nurse - to ask the patient a specific set of questions or to collect specific clinical and laboratory data and then recommend a clinical intervention based on a preprogrammed algorithm [8]. For instance, the app prompts the nurse to collect the patient's blood pressure and to increase the dose of an antihypertensive or refer the patient to a local clinic if the blood pressure exceeds certain predefined thresholds. These apps will play a critical role in CVD management in LMICs for two reasons: by expanding access and affordability because a phone-enabled community health worker can now achieve what would have previously required a visit to a clinician; and by substantially improving healthcare quality through the use of validated clinical algorithms.

The Holy Grail of mHealth is the development of apps that rely on machine-learning algorithms in order to optimize outcomes [9], using clinical and health-systems data to make accurate community- and patient-specific recommendations ('mHealth 3.0'). An example would be an app that recognizes that the patient's blood pressure is elevated and makes recommendations based on the patient's own clinical history, those of similar patients in the community and the real-time availability of healthcare resources. The app then continues to record outcomes and learn from them, thus moving away from the need to hard-code the algorithm. Such apps are increasingly the norm in retail services (e.g., Amazon routinely makes recommendations based on one's previous online purchases and most recent search history), but are rare in healthcare. We anticipate that this will be the area of greatest progress in the coming decade.

It is important to note that despite their enormous potential, there is limited high-quality evidence that mHealth applications have been successfully used to manage CVD in LMICs [10]. Data on their effectiveness currently comes from the literature on HIV (medication reminders may improve short-term antiretroviral adherence [11]) and tobacco cessation (text-based motivation may modestly improve successful quits [12]). With regards to CVD in LMICs, the evidence base is sparse, with a handful of trials of ' $m$ Health 1.0' interventions in middle-income countries with short durations of follow-up $[10,13]$. We urgently need well-designed studies of comprehensive interventions that use large sample sizes and have long-term follow-ups. These studies must examine the impact of $\mathrm{mHealth}$ on multiple healthcare domains, including effectiveness (including long-term patient retention), safety and equity. In order to facilitate the rapid scaling-up of successful interventions after the completion of the study, policy-makers should be engaged early, and process and economic measures should be evaluated alongside clinical outcomes. There are several such trials currently registered at ClinicalTrials.gov, suggesting that the evidence base in support of mHealth is likely to see explosive growth in the coming years.

In addition to the limited evidence of effectiveness, mHealth apps in LMICs face several other challenges. The absence of global technology standards means that a proven intervention may have to be adapted prior to scaling it up to a different setting. Literacy, numeracy and health literacy can be enormous barriers to expanding access to mHealth technologies; in our own work, we have found that less than $30 \%$ of adults in rural India were comfortable with texting [14]. App development must therefore engage with end-users early and frequently, incorporating extensive field testing into the iterative design process. Finally, investigators must be cognizant of evolving regulatory requirements in this space.

In conclusion, we remain optimistic about the potential of mHealth technologies to transform healthcare delivery in LMICs, in part because mobile phones represent ubiquitous and inexpensive computational power and market competition continues to drive down communication costs. Applications that are sensitive to the needs of the end-user and have been validated in clinical trials will fundamentally change the way we delivery healthcare by expanding access, improving healthcare quality and lowering costs. However, additional data are needed in order to define their long-term cost-effectiveness, scalability and sustainability within the existing healthcare infrastructure. Well-designed studies evaluating the long-term clinical and economic impact of advanced mHealth applications should be a research priority, ensuring that mHealth can rise above the rhetoric and deliver on its full potential to help control the CVD epidemic in LMICs.

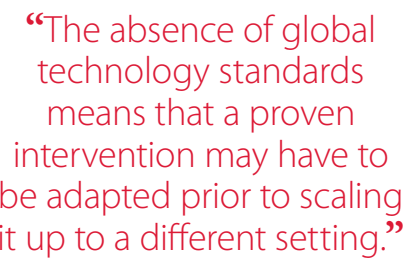
it up to a different setting." 
Financial \& competing interests disclosure The authors have no relevant affiliations or financial involvement with any organization or entity with a financial interest in or financial conflict with the subject matter or materials discussed in the manuscript. This includes employment, consultancies, honoraria, stock ownership or options, expert testimony, grants or patents received or pending, or royalties.

No writing assistance was utilized in the production of this manuscript.

\section{References}

1 Hunter DJ, Reddy KS. Noncommunicable diseases. N. Engl. J. Med. 369(14), 1336-1343 (2013).

2 O’Donnell MJ, Xavier D, Liu L et al. Risk factors for ischaemic and intracerebral haemorrhagic stroke in 22 countries (the INTERSTROKE study): a case-control study. Lancet 376(9735), 112-123 (2010).

3 Yusuf S, Hawken S, Ounpuu S et al. Effect of potentially modifiable risk factors associated with myocardial infarction in 52 countries (the INTERHEART study): case-control study. Lancet 364(9438), 937-952 (2004).

4 Subramanian SV, Corsi DJ, Subramanyam MA, Smith GD. Jumping the gun: the problematic discourse on socioeconomic status and cardiovascular health in India. Int. J. Epidemiol. 42(5), 1410-1426 (2013).

5 The World Bank. Physicians (per 1,000 people) (2013).

http://data.worldbank.org
6 International Telecommunications Union. International Communication Technology Statistics (2014).

www.itu.int

7 Steinhubl SR, Muse ED, Topol EJ. Can mobile health technologies transform health care? JAMA 310 (22), 2395-2396 (2013).

8 Anchala R, Di Angelantonio E, Prabhakaran $\mathrm{D}$, Franco $\mathrm{OH}$. Development and validation of a clinical and computerised decision support system for management of hypertension (DSS-HTN) at a primary health care (PHC) setting. PLoS ONE 8(11), e79638 (2013).

9 Kumar S, Nilsen WJ, Abernethy A et al. Mobile health technology evaluation: the mHealth evidence workshop. Am. J. Prev. Med. 45(2), 228-236 (2013).

10 Beratarrechea A, Lee AG, Willner JM, Jahangir E, Ciapponi A, Rubinstein A. The impact of mobile health interventions on chronic disease outcomes in developing countries: a systematic review. Telemed. J. E. Health 20(1), 75-82 (2014).

11 Horvath T, Azman H, Kennedy GE, Rutherford GW. Mobile phone text messaging for promoting adherence to antiretroviral therapy in patients with HIV infection. Cochrane Database Syst. Rev. 3, CD009756 (2012).

12 Whittaker R, McRobbie H, Bullen C, Borland R, Rodgers A, Gu Y. Mobile phone-based interventions for smoking cessation. Cochrane Database Syst. Rev. 11, CD006611 (2012).

13 Anchala R, Pinto MP, Shroufi A et al. The role of Decision Support System (DSS) in prevention of cardiovascular disease: a systematic review and meta-analysis. PLoS ONE 7(10), e47064 (2012).

14 Kazi DS, Saha P, Mastey N. Mobile phones: hope or hype? A qualitative study of best practices in $\mathrm{m}$-Health development in a low income country. Global Heart 9(Suppl. 1), e312 (2014). 